\title{
Quantitative characterization of interface delamination in a composite T-joint using couplant-free Lamb wave methods
}

Journal of Reinforced Plastics and Composites $0(0) 1-16$

(C) The Author(s) 2015 Reprints and permissions: sagepub.co.uk/journalsPermissions.nav DOI: $10.1177 / 0731684415603233$ jrp.sagepub.com

\author{
Janardhan Padiyar M. and Krishnan Balasubramaniam
}

\begin{abstract}
Need for quick NDE methods during field maintenance of aircraft has become a focus of attention due to increase in use of co-cured skin-stiffened composite structures. When Lamb wave propagates over skin-stiffened structures, the occurrence of multiple modes is unavoidable due to structural features such as stringers and stiffeners, which in turn makes the interpretation of received wave difficult and limits the defect-detection ability of Lamb waves. Using finite element simulations, propagation of incident $S_{0}$ Lamb mode in a typical composite T-joint with delamination between the flange and skin (interface) is investigated. Arrival time delays of the out-of-plane mode-converted wave packets are found to be a promising indicator for quantitative detection and sizing of the delamination. A novel experimental technique by combining a liquid couplant-free transduction scheme using dry-coupled roller transducer (contact probe) for generating $S_{0}$ mode with an air-coupled transducer (non-contact probe) as a receiver is proposed. This transduction scheme helps in selectively separating mode-converted wave packets. This investigation also establishes quantitative B-scan imaging method for characterization of delamination in the T-joint. Experimental result shows a good agreement with finite element predictions. Proposed method being single sided and couplant free has a potential to be utilized for field applications.
\end{abstract}

\section{Keywords}

Stiffened structure, delamination, $S_{0}$ mode, air-coupled ultrasonics, FEM

\section{Introduction}

The stiffened-skin composite structures are an attractive design option for aircraft control surfaces like aileron, elevator, rudder, and flaps. These structures consist of co-cured or co-bonded stiffener such as $\mathrm{T}$, $\mathrm{L}, \mathrm{C}$ and ribs at regular intervals. A typical $\mathrm{T}$-joint or L-joint stiffened composite panel consists of a flange and vertical web that is attached to the skin region to provide bending rigidity to the structure. Orthogonal connections between the flange and the web of T-joint structure are the zone of potential weakness, due to the load transfer taking place in an out-of-plane mode, causing possible zone for delaminations. Sub-surface delamination in these structures may also occur during in-service loads such as due to impact loads or fatigue, which can substantially reduce the strength and stiffness of the structure. However, these delaminations are not visible and accessible for inspection on the surface. Currently, the commonly used ultrasonic NDE techniques for detecting and sizing of defects like delamination in aerospace composite structures are either point-to-point manual A-scan inspection using couplant or automated ultrasonic immersion/ squirter-based C-scan inspection. The conventional squirter and immersion ultrasonic NDE techniques are often not found suitable for field inspection, since they need raster scanners or coupling medium like gel

\footnotetext{
Department of Mechanical Engineering, Center for Nondestructive Evaluation, Indian Institute of Technology Madras, Chennai, India

Corresponding author:

Janardhan Padiyar M, Indian Institute of Technology Madras, Chennai, TN 600036, India.

Email: padiyar@gmail.com
} 
or liquid, which severely limits quick field inspection during maintenance. Therefore, there is a need for quick non-destructive approaches to evaluate delamination-type damage to assess the structure's condition.

Lamb waves are guided waves that propagate in thin plate-like structures and have been found suitable for detection of defects over a large area quickly compared to conventional ultrasonic bulk wave inspection. Several studies have examined Lamb waves as a means for damage identification in composites and various techniques for damage characterization have been proposed. ${ }^{1}$ However, when a Lamb wave propagates over a skin-stiffened structure the occurrence of multiple Lamb modes becomes inevitable due to presence of geometrical discontinuity, which is inherently present due to structural requirements. ${ }^{2-4}$ Furthermore, flange delaminations act as thickness change discontinuities, producing mode conversion and generating different wave packets. Additionally, Lamb wave propagation suffers high attenuation in composite material due to intrinsic material damping and extrinsic damping due to structural geometries like stiffener, ribs, and ply drops. These issues make the interpretation of received Lamb wave signal difficult and sizing the damage can be challenging. Many studies have investigated different delamination detection techniques using fundamental anti-symmetric Lamb wave $\left(\mathrm{A}_{0}\right)$ mode $^{5-12}$ and fundamental symmetric Lamb wave $\left(\mathrm{S}_{0}\right)$ mode $^{13-17}$ in flat composite laminates, repair patch, ${ }^{18}$ beams ${ }^{19}$ using FE simulation, and experiments. Correspondingly, various signal features ranging from signal attenuations, arrival time, reflected wave, or scattered by the delamination have been examined to identify and characterize delamination. ${ }^{20-22}$ To transmit and receive Lamb waves in composites, different types of contact and non-contact ultrasonic transducers have been utilized such as wedge-mounted transducer, drycoupled, air-coupled, laser ultrasonics, piezoelectric wafer-active sensors, ${ }^{23,24}$ or a combination of these transducers. Other damage detection studies have been performed in composite structures using optical detection methods such as Fibre Bragg grating sensors for detecting Lamb wave signal and laser ultrasonics for observing full-field Lamb wave propagation characteristics with different defects. ${ }^{25,26}$ Laser ultrasound poses some practical problems such as cost, difficulty in selective reception of a particular Lamb mode, bandwidth of the Lamb waves generated, dependency of signal-to-noise ratio on reflectivity of the surface, and issues with laser safety. Among the various ultrasonic transducers, air-coupled ultrasonic transducer can comfortably generate and detect Lamb waves in acoustically matching composites. Due to couplant free, noncontact nature, and selective reception/transduction of the Lamb wave, they have been successfully used for delamination characterization studies. ${ }^{5-11}$

Although there are some studies that examine effect of delamination on Lamb wave propagation in flat laminates, the effect of geometrical discontinuity particularly in combination with delamination on $\mathrm{S}_{0} \mathrm{Lamb}$ wave propagation in skin-stiffened structure like T-joints has rarely been addressed. Even though a realistic approach of Lamb wave-based inspection is to analyze mode-converted wave packets as signature for delamination, this topic has remained a challenging task due to interference of wave modes, overlapping of the signal from the edges, dispersion, and multimode nature of the Lamb waves. Thus, the aim and uniqueness of this study was to investigate a specific problem of $\mathrm{S}_{0}$ mode wave propagation in the composite $\mathrm{T}$-joint stiffened structure using numerical modelling to highlight the complex interaction and mode conversion of $\mathrm{S}_{0}$ mode with a geometrical discontinuity and delamination. Additionally, using a liquid couplant-free transduction of $\mathrm{S}_{0}$ mode and selective reception of the specific mode-converted wave packets, this study proposes two methods for detection and sizing of interface delamination in the T-joint using a (a) arrival timebased and (b) B-scan-based imaging methods. The following sections describe $S_{0}$ mode transduction and mode conversions across the web of the $\mathrm{T}$-joint with different sizes of interface delaminations using numerical simulations, followed by comparison with experimental measurements. This study will also explore the use of B-scan imaging technique for quantitative characterization of interface delamination using simulation and experiments and followed by discussion of the results.

\section{Transduction of $S_{0}$ mode}

It is usually desirable that Lamb wave excitation in the structure is at a low frequency-thickness product region of the dispersion curves. This kind of excitation ensures that only two fundamental Lamb wave modes $\mathrm{S}_{0}$ and $\mathrm{A}_{0}$, which are relatively non-dispersive, propagate in the structure. There are two common transducer configurations for the transduction of Lamb waves, the pulse-echo and pitch-catch configuration. Studies have reported delamination detection by examining the reflected Lamb waves from the delamination in pulse-echo transduction configuration ${ }^{13-16}$; while in a pitch-catch configuration, transmitted Lamb waves have been analyzed for delamination detection. ${ }^{5-11}$ However due to the higher attenuation of Lamb waves in composite material, a pitch-catch is preferred compared to pulse-echo configuration. The $\mathrm{S}_{0}$ mode propagates much longer distance than $\mathrm{A}_{0}$ mode in 
composites due to its lower attenuation coefficient. ${ }^{27}$ Moreover, in the low frequency-thickness region, advantages of $\mathrm{S}_{0}$ mode are its higher velocity than $\mathrm{A}_{0}$ mode and its energy distribution, which is relatively uniform throughout the structure thickness. The wedge-based $\mathrm{S}_{0}$ mode excitation requires liquid couplant (typically liquid or gel). The angle of the wedge also depends on the material property of composite structure and may need frequent changes in angle of the wedge for reception of specific Lamb mode. Additionally, traversing the wedge during automated scanning with constant layer of liquid couplant becomes a challenge.

Non-contact excitation and reception of Lamb waves using either laser ultrasonics or air-coupled transducers are being increasingly used. In early works, visualization of propagating Lamb waves across the impact damages using a scanning laserDoppler vibrometry ${ }^{28}$ and air-coupled ultrasonic transducers ${ }^{29,30}$ was proposed to image the scattered out-ofplane Lamb wave field in plate-like structures. In recent work, Hillger et al. ${ }^{31,32}$ presented an application more directly related to the present paper. The PZT transducers as transmitter were glued on a section of an EC135 helicopter tail-boom structure, and $\mathrm{S}_{0}$ mode propagation and mode conversion to $\mathrm{A}_{0}$ at the impact damage were monitored using automated air-coupled ultrasonic scanning. They used B-scan images to reveal different mode conversions in the vicinity of the impact damage. However, selective mode conversion of the Lamb waves was not achieved and operation at lower frequencies caused overlapping of the $\mathrm{S}_{0}$ and $\mathrm{A}_{0}$ transmitted and mode-converted modes. Generation and reception of Lamb waves by aircoupled transducers is relatively economical compared to laser ultrasonics and an excellent compromise since the reception of waves being independent of reflectivity of the surface. However, preliminary in-house studies showed that air-coupled transducers could not generate $\mathrm{S}_{0}$ mode of sufficient amplitude in composite material, despite the fact they are sensitive to out-of-plane component of $\mathrm{S}_{0}$ mode and $\mathrm{A}_{0}$ mode. To overcome this difficulty, a liquid couplant-free transduction using dry coupled ultrasonic roller transducer as a transmitter for excitation of the $\mathrm{S}_{0}$ mode is proposed. Since drycoupled transducer generates both $\mathrm{S}_{0}$ and $\mathrm{A}_{0}$ mode, a novel temporary couplant-free fixed source and traversing air-coupled transducer as a receiver in pitch-catch configuration is proposed in this study. This novel transduction configuration aids in separation of $\mathrm{S}_{0}$ and $\mathrm{A}_{0}$ Lamb modes in time domain, facilitates controlled single mode interaction with the delamination, selective reception of the $\mathrm{A}_{0}$ or $\mathrm{S}_{0}$ mode, faster scanning, and repeatable measurements. Thus, a targeted Lamb wave mode conversion can be achieved.

\section{Numerical simulation of Lamb wave propagation in a T-joint}

The interaction and propagation of Lamb waves in the stiffened structure is complex and hence an effective numerical method is required to understand this interaction. Finite element numerical simulations of wave propagation in a composite $\mathrm{T}$-joint were proposed to study the interaction of $\mathrm{S}_{0}$ Lamb mode with the geometrical discontinuity, delamination and understand the mode conversion phenomena. Figure 1(a) shows the schematic of a typical composite $\mathrm{T}$-joint and its layup used in aerospace structural applications. In a T-joint, the vertical element is termed the web, and the two horizontal elements are termed flanges. The upper skin and the flange form the thick region and the other side of the upper skin itself forms the thin region. The interface between the upper skin region and the flange has a high affinity for failures in delamination mode, when the joint is subjected to external loads. We term this failure as 'interface delamination'. The interface delamination divides the thick region of the skin of the T-joint locally into two sub-laminates of layup $(0 / 90 / 90 / 0)$. To study the propagation of Lamb waves in a structure, we require the computation of dispersion curves, which inform about the different modes that can propagate in the structure at different frequencies. However, at low frequency-thickness product, $\mathrm{S}_{0}$ and $\mathrm{A}_{0}$ modes are the only Lamb modes that can propagate in the $\mathrm{T}$-joint structure. A centre frequency of $200 \mathrm{kHz}$ was chosen as frequency of Lamb wave excitation, so that excitation is within the low frequency-thickness region of the dispersion curve and due to availability of both roller transducer and aircoupled transducer of $200 \mathrm{kHz}$ during experiments. The material of the T-joint was GFRP, and its properties are listed in Table 1. Table 2 gives the theoretical group velocities of the $\mathrm{S}_{0}$ and $\mathrm{A}_{0}$ modes calculated from Disperse $^{\mathrm{TM}}$ in the thin and the thick regions of the T-joint at a frequency of $200 \mathrm{kHz} .^{33}$

\section{Coupled acoustic-elastic FE model}

To simulate the wave propagation in the T-joint along with the reception of bulk waves in air by an aircoupled receiver, an acoustic-structural coupled 2D plane-strain FE model comprising of both elastic medium (solid) in contact with acoustic medium (fluid) is proposed. Numerical simulation of transient wave propagation was performed using FE package ABAQUS/Explicit $6.9^{\circledR}$. The geometry of the T-joint model used in simulation is shown in Figure 1(a). Taking the $\mathrm{Y}$-axis as reference in Figure 1(a), the layup of the T-joint in the thick, thin, and the web 


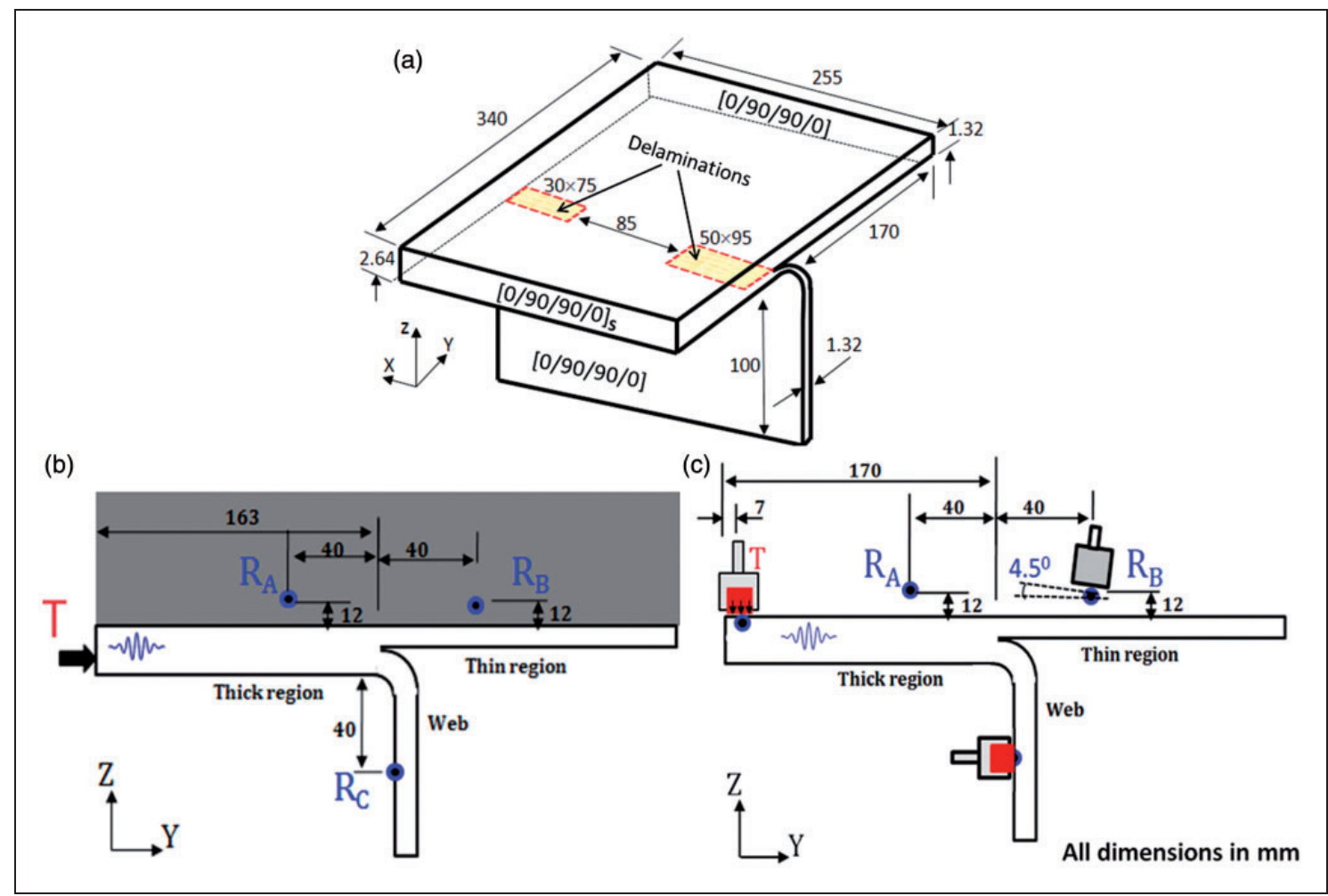

Figure I. (a) Schematic geometry of the composite T-joint with an interface delamination. The locations of the transmitter and receiver on the T-joint for capturing A-scan in the (b) numerical model and (c) experiments.

Table I. Material properties of glass/epoxy lamina.

\begin{tabular}{ll}
\hline Material properties & \\
\hline Density $\rho\left(\mathrm{kg} / \mathrm{m}^{3}\right)$ & 1850 \\
Longitudinal modulus, $\mathrm{E}_{11}(\mathrm{GPa})$ & 46 \\
Transverse modulus, $\mathrm{E}_{22}(\mathrm{GPa})$ & 8.7 \\
In-plane shear modulus, $\mathrm{G}_{12}(\mathrm{GPa})$ & 3.2 \\
Out-of-plane shear modulus, $\mathrm{G}_{23}(\mathrm{GPa})$ & 3.2 \\
Major Poisson's ratio, $v_{12}$ & 0.28 \\
Out-of-plane Poisson's ratio, $v_{23}$ & 0.36
\end{tabular}

regions was $[0 / 90 / 90 / 0]_{\mathrm{S}},[0 / 90 / 90 / 0]$, and $[0 / 90 / 90 / 0]$, respectively. The thickness of each lamina was $0.33 \mathrm{~mm}$. Since the number of plies in each region was known, thickness of the web and the thin region worked out to $1.32 \mathrm{~mm}$ each and that of the thick region to $2.64 \mathrm{~mm}$. Each ply was modelled separately with its corresponding material properties. The surfacebased *TIE constraint in ABAQUS was used to join surfaces of individual composite layers in the model. Tie constraints tie two separate surfaces together so that there is no relative motion between them. The nodes on the slave surface are constrained to have the
Table 2. Theoretical group velocities of the $S_{0}$ and $A_{0}$ modes in the thick and the thin region from the Disperse ${ }^{T M}$ program.

\begin{tabular}{lllll}
\hline Layup & $\begin{array}{l}\mathrm{S}_{0} \text { mode } \\
(\mathrm{m} / \mathrm{s})\end{array}$ & $\begin{array}{l}\mathrm{A}_{0} \text { mode } \\
(\mathrm{m} / \mathrm{s})\end{array}$ & $\begin{array}{l}\mathrm{S}_{0} \text { reception } \\
\text { angle } \theta\left(^{\circ}\right)\end{array}$ & $\begin{array}{l}\mathrm{A}_{0} \text { reception } \\
\text { angle } \theta\left(^{\circ}\right)\end{array}$ \\
\hline$[0 / 90 / 90 / 0]_{\mathrm{S}}$ & 3669 & 1339 & 17 & 5 \\
{$[0 / 90 / 90 / 0]$} & 3749 & 1342 & 18 & 4.5 \\
\hline
\end{tabular}

same value of displacement, as the point on the master surface to which they are tied. The interface delamination was modelled by disconnecting the surfaces of adjacent layers, i.e. by removing *TIE constraint at the delamination region. The surface-based constraints use master-slave formulations where one surface is assigned as a master and other surface as a slave. The acoustic-structural coupling between the acoustic domain and the structural domain at the interface was also achieved by using the *TIE constraints in ABAQUS. On such an acoustic-structural boundary, the acoustic and structural elements have the same displacement normal to the boundary, but the tangential motions are uncoupled. In this study, the acoustic domain in contact with structural domain was assigned as the master surface and the top surface of the 
composite laminate as the slave surface. The model was discretized using 2D plane strain element, CPE4R in the elastic medium. The inviscid fluid medium (air) on top of the T-joint was modelled using acoustic element AC2D4R (pressure field), four node 2D acoustic quadrilateral element having pressure as degree of freedom at each node. For the convergence and the accuracy of the dynamic response in the numerical solution, the selection of stable time step is based on CourantFriedrichs-Lewy (CFL) criteria. The stable time step chosen must be less than the time taken for the fastest wave to travel between two adjacent nodes in the mesh. The recommended time step is $\Delta t=1 /\left(20 \mathrm{f}_{\max }\right)$ where $\mathrm{f}_{\max }$ is the maximum frequency of the dynamic problem and element size must be less than 1/20 times the shortest wavelength. ${ }^{34}$ At the excitation frequency of $200 \mathrm{kHz}$ the $\mathrm{A}_{0}$ mode has the lowest velocity and the shortest wavelength in the elastic domain. The velocity of sound in air is $344 \mathrm{~m} / \mathrm{s}$ at room temperature, which is lower than $\mathrm{A}_{0}$ mode. Hence, the wave having the shortest wavelength is in the acoustic domain. The mesh size was chosen closer to 20 nodes per wavelength in the acoustic domain, which in turn results in about 80 elements per shortest wavelength in the elastic domain. The element size of $0.1 \mathrm{~mm} \times 0.1 \mathrm{~mm}$ is assigned to the mesh, which satisfies the CFL criteria in both the domains. Such a mesh density leads to approximately 0.9 million elements in the acoustic and elastic domain in total. Mapped meshing was used to generate a uniform mesh size in the straight and curved portions of the T-joint, since this type of meshing provides minimum distortion of elements. Even though dry-coupled transducer generates both $\mathrm{S}_{0}$ and $\mathrm{A}_{0}$ modes in experiment, only normalized displacements of $S_{0}$ mode were imposed on the right edge of the mesh in the FE model. Receivers were deployed in the form of a set of point receivers in the acoustic medium. The locations of the transmitter and receiver in the FE model are shown in Figure 1(b). The input excitation waveform at the transmitter was $200 \mathrm{kHz}$ with seven cycles tone burst, modulated using Hanning window. The time integration was performed using through a central difference scheme in ABAQUSExplicit with a time step of $5.72 \mathrm{~ns}$. The pressure field in the acoustic medium and the displacement field in the T-joint were computed at each time step. Output from the FE model can be a pressure-time history in the fluid medium and displacement-time history in the solid medium. The propagating wave in both the domains can also be observed using contour plots. For visualization of wave leakage into air, the acoustic medium can be modelled on all the sides of the T-joint. However to avoid need of high computational resources, the acoustic medium needs to be present only over the skin region of $\mathrm{T}$-joint.

\section{Interaction of $\mathrm{S}_{0}$ Lamb mode with geometrical discontinuity in T-joint}

Two sets of numerical simulations were carried out by interchanging the positions of the transmitter and receiver with respect to the thick and the thin regions in the $\mathrm{T}$-joint. In the first case, the transmitter and receiver were deployed at the locations as shown in Figure 1(b). The symmetric Lamb mode $\left(\mathrm{S}_{0}\right)$ was excited by giving both normalized in-plane and out-of-plane displacements components from displacement profile calculated from Disperse ${ }^{\mathrm{TM}}$ as boundary conditions. Receivers $R_{A}$, and $R_{B}$ were positioned above the elastic medium, i.e. in the acoustic medium to simulate the air-coupled ultrasonic receiver positions as shown in Figure 1(c). However, web region is generally not accessible in a real structure; for the sake of completeness, a receiver $R_{C}$ is positioned on the web region in the elastic domain that captures out-of-plane displacement time history. It is proposed here to compare the arrival time of each wave packets of the A-scans from numerical simulation model with the arrival time computed using theoretical group velocities of wave packets ( $\mathrm{S}_{0}$ and $\mathrm{A}_{0}$ modes) propagating in the $\mathrm{T}$-joint. For each A-scan from the numerical simulation, an envelope of a Hilbert transform was taken and the ToF of the peak envelope of the each wave packet was used as the time for computation of the group velocity and the particular wave mode was identified. Here, the focus of this study was on the peak of the first two wave packets in the A-scan; the latter signals if any were time gated.

\section{Transmitter on the thick region}

In this case, $\mathrm{S}_{0}$ mode propagates and interacts with the front edge of the delamination and undergoes mode conversion. In this configuration shown in Figure 1(b) at the receiver $R_{B}$, the time of arrival of the first two wave packets from numerical A-scan shown in Figure 2(a) was 90 and $135.4 \mu$ s, respectively. At the receiver $R_{B}$, the time taken for the arrival of the incident $S_{0}$ mode from the point of excitation computed using the theoretical group velocities from Table 2 is $90.2 \mu \mathrm{s}$, which matches well with the numerical A-scan. Thus, we can conclude that the first wave packet is of $\mathrm{S}_{0}$ mode as it travels from the point of excitation to the point of reception. The transmitted $\mathrm{S}_{0}$ mode across the discontinuity is denoted by $\mathrm{S}_{0} \mathrm{~S}_{0}$ wave packet where the first letter indicates the source mode, while the second letter indicates the current propagating mode (this notation is used throughout this paper). By similar analogy, the second wave packet denoted by $\mathrm{S}_{0} \mathrm{~A}_{0}$ in Figure 2(b), which corresponds to the mode-converted $\mathrm{A}_{0}$ mode due to interaction of the incident $\mathrm{S}_{0}$ mode with the geometrical discontinuity. The time of arrival 


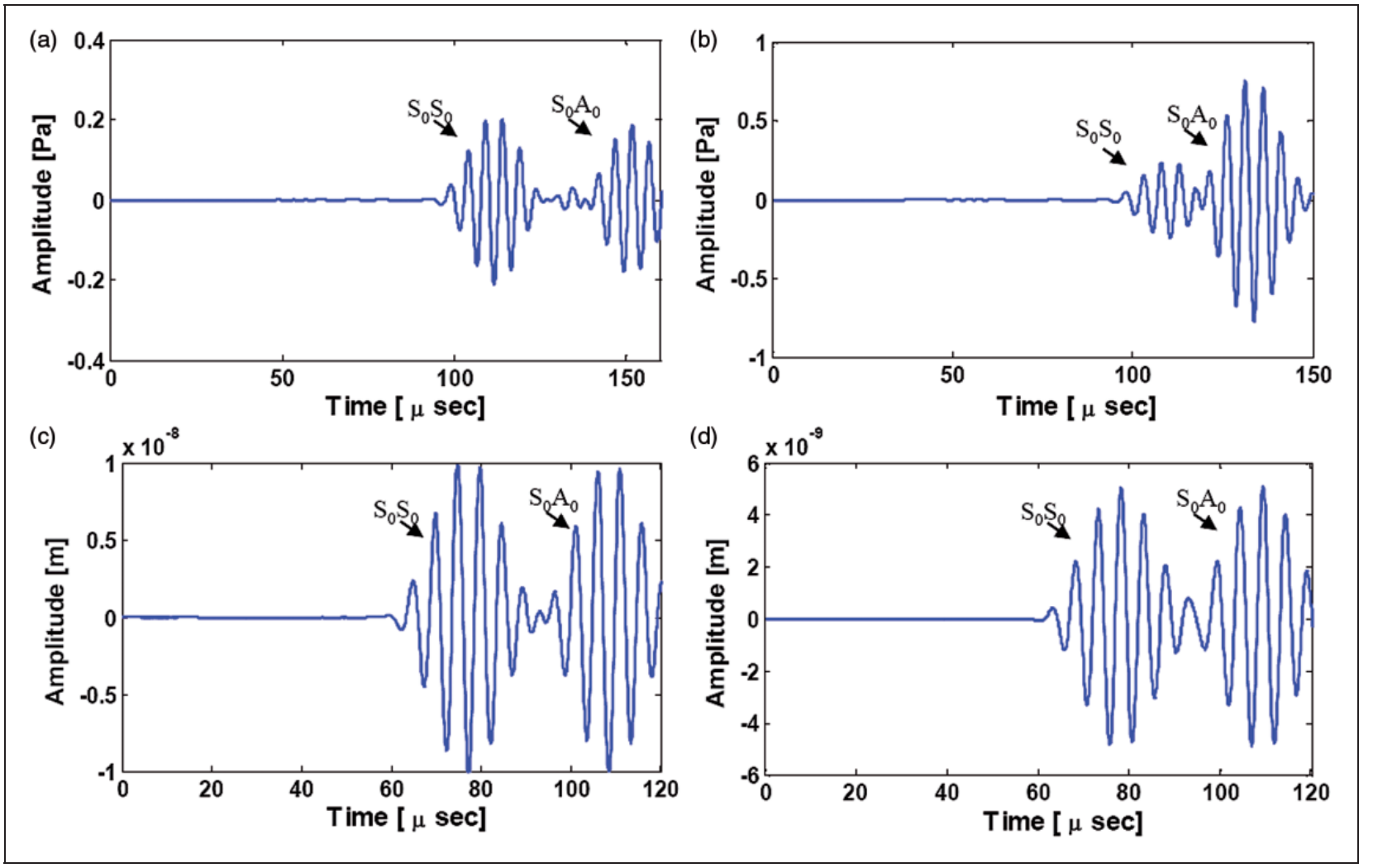

Figure 2. Pressure-time trace from numerical simulations captured (a) at $R_{B}$ when the transmitter is on the thick region, receiver at $40 \mathrm{~mm}$ from centre of the T-joint on the thin region, (b) at $R_{A}$ when the transmitter is on the thin region, receiver on the thick region at $40 \mathrm{~mm}$ from the centre of the T-joint. Out-of-plane displacement component at receiver $R_{C}$ on the web region from numerical simulation (c) when the transmitter is on the thick region (d) when the transmitter is on the thin region.

of the second wave packet from the A-scan shown in Figure 2(b) was $135.4 \mu \mathrm{s}$, which matches well when compared with the computed time of arrival of $\mathrm{S}_{0} \mathrm{~A}_{0}$ wave packet of $135 \mu$ s using the theoretical group velocities.

\section{Transmitter on the thin region}

In this configuration, A-scan recorded at the receiver $R_{A}$ is shown in Figure 1(b). The time of arrival of first two wave packets from the numerical A-scan was 90.6 and $130.4 \mu \mathrm{s}$, respectively. At the receiver $\mathrm{R}_{\mathrm{A}}$, the time taken for the arrival of the incident $\mathrm{S}_{0}$ mode from the point of excitation computed using the theoretical group velocities listed in Table 2 is $89.6 \mu$ s, which matches well with the computed time of $89.4 \mu \mathrm{s}$. Thus, we can conclude that the first wave packet is $\mathrm{S}_{0} \mathrm{~S}_{0}$ wave packet. The second wave packet from the A-scan shown in Figure 2(b) was having time of arrival of $132 \mu \mathrm{s}$, which is closely matching with $135 \mu \mathrm{s}$, time of arrival computed from theoretical group velocities of $S_{0}$ and $\mathrm{A}_{0}$ mode shown in Table 2 . Thus, it can be confirmed that the second wave packet is $\mathrm{S}_{0} \mathrm{~A}_{0}$.

\section{Receiver on the web region}

From the previous numerical analysis, it is apparent that when the $\mathrm{S}_{0}$ mode interacts with structural discontinuity, mode conversion takes place and $\mathrm{A}_{0}$ mode is generated. This generated $A_{0}$ mode with the incident $S_{0}$ also propagates into the web region. The A-scan shown in Figure 2(c) is captured at the location $R_{C}$ when the transmitter on the thick region and receiver on the web region. Similarly, when the transmitter is on the thin region and receiver on the web region, the A-scan captured at $R_{C}$ is shown in Figure 2(d). With similar analogy as discussed in the previous two sections, the time of arrival of wave packets received in the web region at the location $R_{C}$ is calculated and wave packets are identified. The arrival time of different wave packets obtained by the FE method for different configurations is tabulated in Table 3, which shows a fairly good approximation. The numerical model can also be used for visualization of Lamb wave leakage into the acoustic medium. This visualization may also help in identifying complex conversion taking place in the T-joint with different geometries. Figure 3 shows snapshots of 
Table 3. Comparison of wave packets arrival time from numerical A-scans with estimated values at the receiver location shown in Figure 2.

\begin{tabular}{|c|c|c|c|c|c|}
\hline $\begin{array}{l}\text { Transmitter } \\
\text { location at } \\
\text { the edge }\end{array}$ & $\begin{array}{l}\text { Receiver } \\
\text { location }\end{array}$ & $\begin{array}{l}\text { Estimated } \\
\text { arrival time } \\
\text { of wave packet } \\
\mathrm{S}_{0} \mathrm{~S}_{0} \text { Disperse }{ }^{\mathrm{TM}} \mu \mathrm{s}\end{array}$ & $\begin{array}{l}\text { Estimated arrival } \\
\text { time of wave packet } \\
\mathrm{S}_{0} \mathrm{~A}_{0} \text { Disperse }{ }^{\mathrm{TM}} \mu \mathrm{s}\end{array}$ & $\begin{array}{l}\text { Arrival time of first } \\
\text { wave packet from } \\
\text { simulation } \mu \mathrm{s}( \pm 0.4 \mu \mathrm{s})\end{array}$ & $\begin{array}{l}\text { Arrival time of } \\
\text { second wave packet } \\
\text { from simulation } \\
\mu \mathrm{s}( \pm 0.4 \mu \mathrm{s})\end{array}$ \\
\hline Thick region & $\mathrm{R}_{\mathrm{B}}$ & 90.2 & 135 & 90 & 135.4 \\
\hline Thick region & $\mathrm{R}_{\mathrm{C}}$ & 55.1 & 92.2 & 56 & 92 \\
\hline Thin region & $\mathrm{R}_{\mathrm{A}}$ & 89.4 & 134.8 & 90.6 & 132 \\
\hline Thin region & $\mathrm{R}_{\mathrm{C}}$ & 54.2 & 93.1 & 55 & 93 \\
\hline
\end{tabular}

the $\mathrm{S}_{0}$ mode propagating from thin to thick region at different instances of time in the acoustic domain over the T-joint, when the transmitter is at the thin region. Mode conversion and propagation of different wave packets at the delamination site (marked with arrows) can be observed in addition to the transmitted and reflected wave. Furthermore, from the snapshots, we can observe that the incident mode along with modeconverted wave packets travels into the web region. Thus, from the numerical simulation we can conclude that, when the $S_{0}$ mode propagates from the thick to the thin region or vice versa, $S_{0}$ mode is converted to $\mathrm{S}_{0} \mathrm{~S}_{0}$ and $\mathrm{S}_{0} \mathrm{~A}_{0}$ wave packets and these wave packets can be used as feature for delamination identification.

\section{Interaction of $S_{0}$ Lamb mode with the interface delamination}

Due to interface delamination in the T-joint there is a shift in the location of the geometrical discontinuity when compared with the geometry of the healthy T-joint. Consequently, when there is an interface delamination, the distance of wave propagation in the thin and thick region changes when compared to a healthy T-joint and finally results in the time shift of the wave packets captured at a particular point. This time shift was used to size the interface delamination, which forms a basis for the present work.

\section{Sizing of delamination by time-of-arrival (ToA) of $S_{0} A_{0}$ wave packet}

In the numerical model of the T-joint, an interface delamination near the centre of the web region was introduced by disconnecting the surfaces of adjacent layers, i.e. by removing *TIE constraint at the delamination region. The region without delamination was connected to the adjacent layers by using *TIE constraint thus ensuring continuity of displacements at the interface. The excitation and boundary conditions are same as discussed earlier. The transmitter and receiver were positioned over the thin and thick regions, respectively, as shown in Figure 1(b). Let $L_{l}$ denote the horizontal distance between the transmitter on the right edge of the T-joint and the edge of interface delamination (i.e. thin region) and let $L_{2}$ denote the horizontal distance between the receiver and start of interface delamination. ' $D$ ' denotes the interface delamination width. Thus, the distance between the transmitter and receiver is $L_{1}+D+L_{2}$ as shown in Figure 4(b). The arrival time of the generated $\mathrm{S}_{0} \mathrm{~A}_{0}$ wave packet at the receiver $\mathrm{R}_{1}$ due to mode conversion at the geometrical discontinuity can be expressed as follows

$$
t_{o}=\frac{L_{2}+D}{V_{A_{0} \text { Thick }}}+\frac{L_{1}}{V_{S_{0} \text { Thin }}}
$$

where $V_{\mathrm{S}_{0} \text { Thin }}$ and $V_{\mathrm{A}_{0} \text { Thick }}$ are group velocities of the $\mathrm{S}_{0}$ and $\mathrm{A}_{0}$ mode in the thin and thick regions, respectively. If there is no interface delamination, the thick region spreads up to the centre of the T-joint until the web, and the size of the thick region becomes $L_{l}+D$. The arrival time of $\mathrm{S}_{0} \mathrm{~A}_{0}$ wave packet at the receiver is then given by the following equation

$$
t_{o}=\frac{L_{2}+D}{V_{A_{0}} \text { Thick }}+\frac{L_{1}}{V_{S_{0} \text { Thin }}}
$$

Subtracting equation (2) from (1), the following equation is obtained

$$
\Delta t=t_{1}-t_{0}=D\left(\frac{1}{V_{S_{0} \text { Thin }}}-\frac{1}{V_{A_{0} \text { Thick }}}\right)
$$

From expression (3), the difference in arrival times of $\mathrm{A}_{0}$ mode and the group velocities in the thick and thin regions are known, the width of interface delamination $(D)$ can be estimated. Four different sizes of interface delaminations, i.e. healthy, 40, 50, and $60 \mathrm{~mm}$ were simulated and analyzed in the numerical study. A-scan obtained at healthy region of the T-joint is shown in Figure 5(a). A-scans obtained for each case of the interface delamination are shown in Figure 5(b) to (d). From the numerical A-scans in Figure 5(b) to (c), it was observed that when the delamination size 


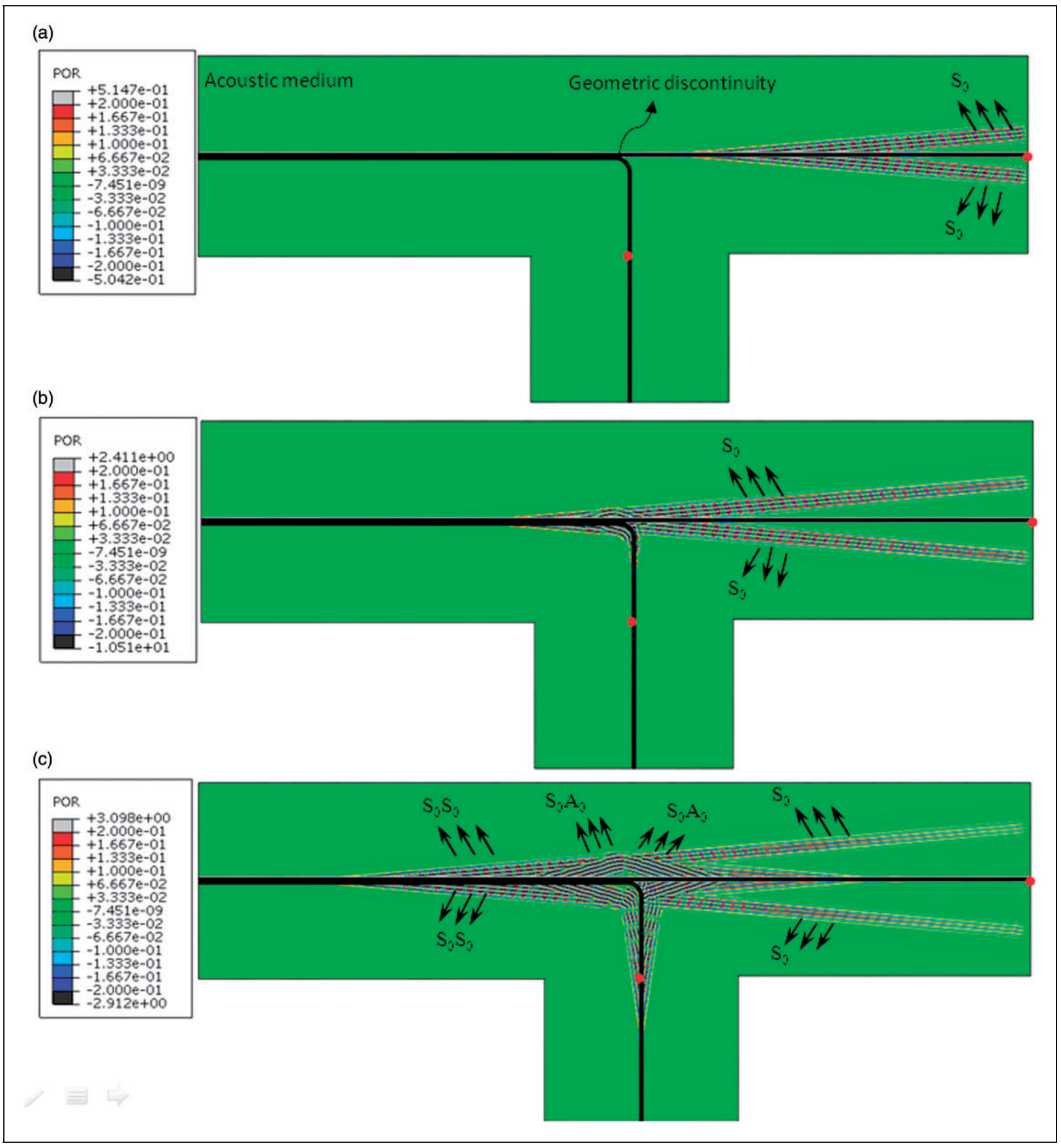

Figure 3. $\mathrm{S}_{0}$ mode interaction with geometrical discontinuity in a T-joint and wave propagating from the right edge (thin region) to the left edge (thick region) in a 2D FE model. Snapshots of pressure contours at different instances of time observed in the acoustic domain around the T-joint (a) $45 \mu \mathrm{s}$, (b) $65 \mu \mathrm{s}$, (c) $85 \mu$ s showing mode conversion to $\mathrm{S}_{0} \mathrm{~S}_{0}$ and $\mathrm{S}_{0} \mathrm{~A}_{0}$ wave packets; arrows indicate the leakage direction of the wave mode. Slopes of the leaking planar wave give information of the type of Lamb mode. (Acoustic domain is $50 \mathrm{~mm}$ on both the sides of the T-joint.)

increases the relative separation between wave packets $\mathrm{S}_{0} \mathrm{~S}_{0}$ and $\mathrm{S}_{0} \mathrm{~A}_{0}$ decreases. In Figure 5(c), wave packets $\mathrm{S}_{0} \mathrm{~S}_{0}$ and $\mathrm{S}_{0} \mathrm{~A}_{0}$ interfere partially. Moreover, in the A-scan of $60 \mathrm{~mm}$ interface delamination shown in Figure5(c), the wave packets $\mathrm{S}_{0} \mathrm{~S}_{0}$ and $\mathrm{S}_{0} \mathrm{~A}_{0}$ interfere fully with each other.

\section{Experimental validation}

In the experimental work, $\mathrm{S}_{0}$ wave propagation in a T-joint structure having geometric length scale similar to those used in simulations was investigated. Here we compare the arrival times of wave packets in the 


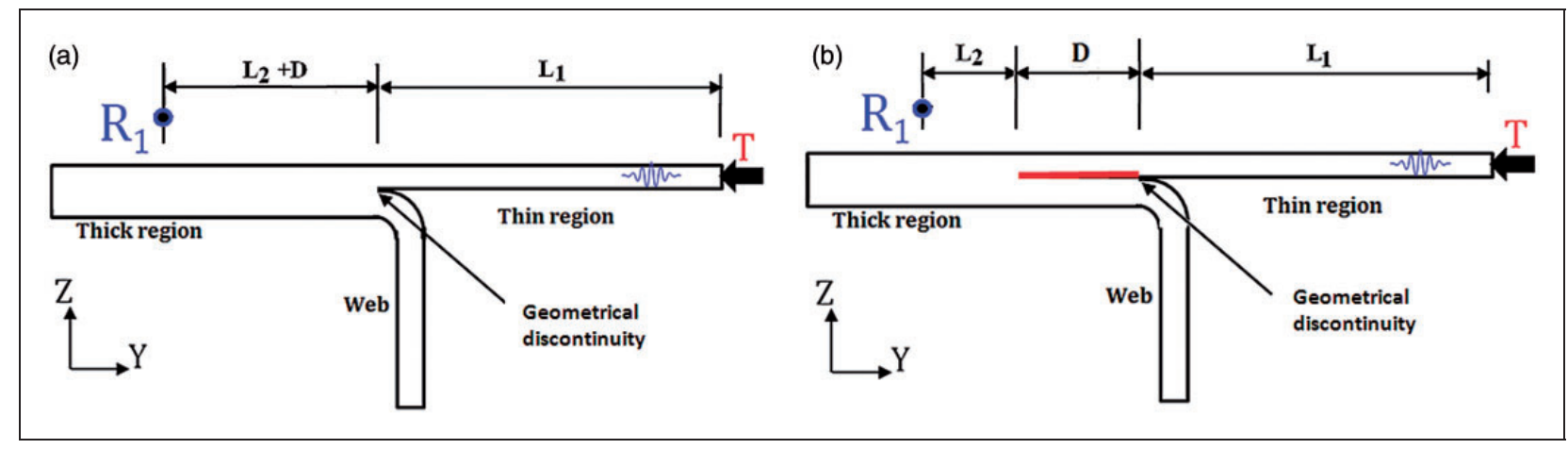

Figure 4. Schematic of locations of the transmitter and receiver used in numerical model of T-joint (a) without delamination (b) with delamination.

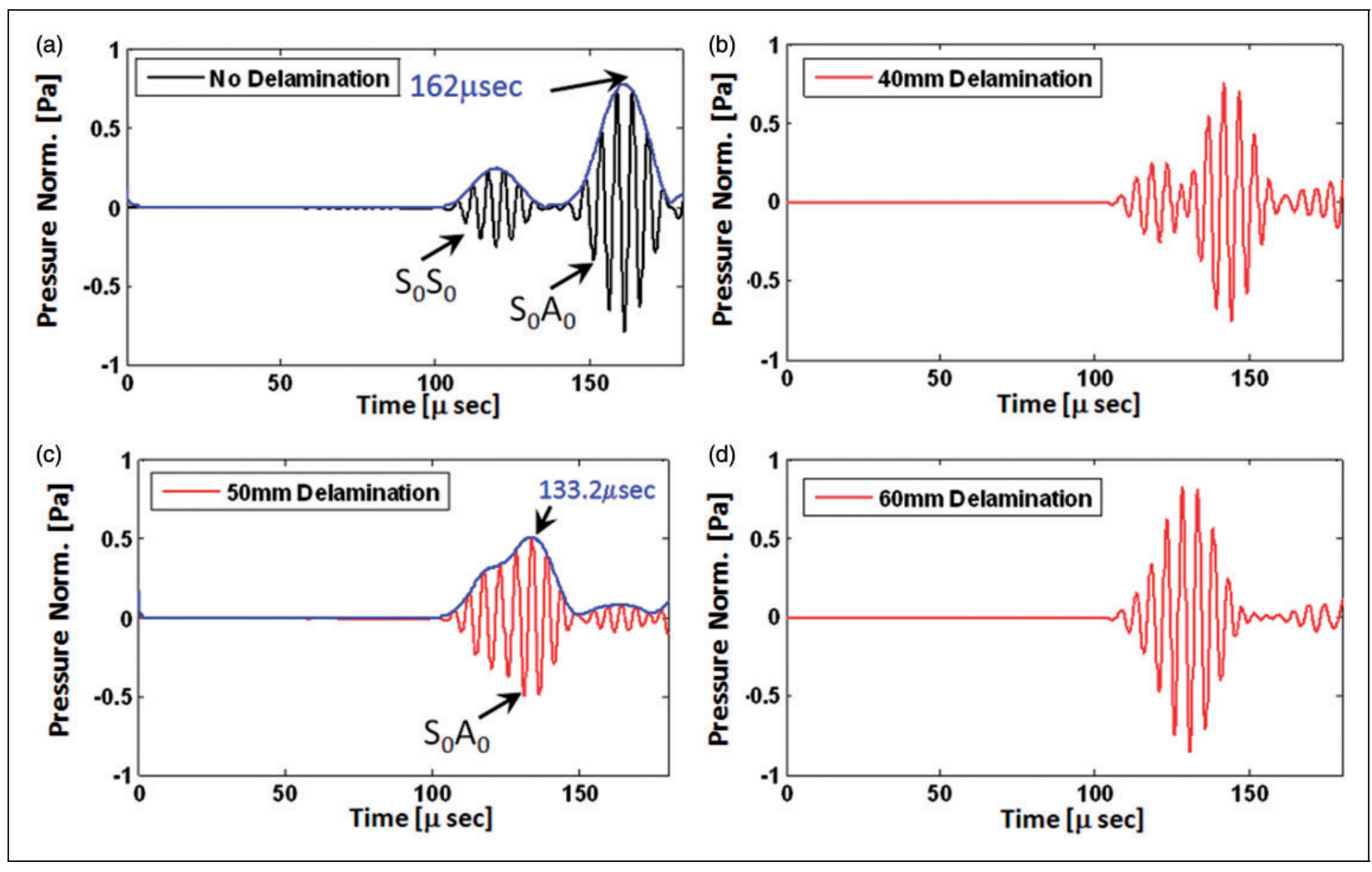

Figure 5. A-scans from the numerical model of the T-joint, in the (a) healthy region and at the interface of delamination of widths (b) $40 \mathrm{~mm}$, (c) $50 \mathrm{~mm}$, (d) $60 \mathrm{~mm}$ captured at a distance of $75 \mathrm{~mm}$ from the web location in thick region.

experimental waveform captured by the air-coupled receiver to that of numerical A-scans. The experimental setup consisted of a pulser-receiver to excite the roller transducer, $100 \mathrm{MHz} \mathrm{A} / \mathrm{D}$ card, motion controller, $\mathrm{x}-\mathrm{y}$ scanner for moving the air-coupled transducer, and a laptop for data storage. For Lamb wave generation and detection in a pitch-catch arrangement, a dry-coupled roller transducer from SonaTest ${ }^{\mathrm{TM}}$ is used as a transmitter and air-coupled ultrasonic receiver with a resonant frequency of $200 \mathrm{kHz}$ from Ultran Group. Transducer-holding fixtures were fabricated to hold air-coupled receiver and dry-coupled roller transducer. The transducers were mounted on an $\mathrm{x}-\mathrm{y}$ scanner and a constant elevation was maintained between the aircoupled receiver and the surface of $\mathrm{T}$-joint specimen during translation. The transmitter and receiver unit was positioned at the either side of the web region as shown in Figure 1(c). The angle of the air-coupled 


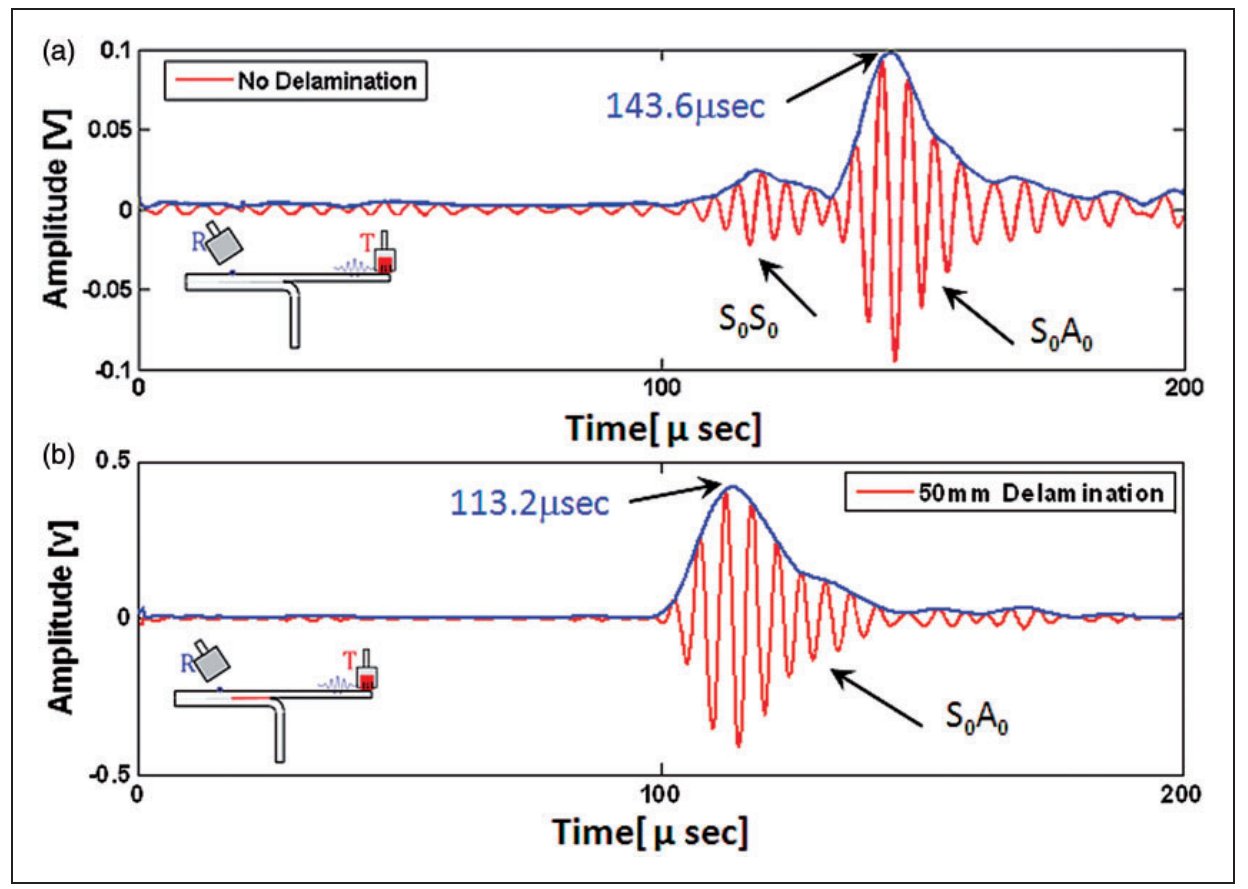

Figure 6. Experimental A-scans from (a) healthy and (b) $50 \mathrm{~mm}$ width interface delamination regions in a T-joint.

receiver (facing forward) can be adjusted to receive either $\mathrm{S}_{0}$ or $\mathrm{A}_{0}$ mode. The number of cycles in the excitation pulse was seven, which corresponds to $35 \mu \mathrm{s}$. The transducer unit was positioned over the healthy region and A-scan shown in Figure 6(a) was captured and saved in this configuration. This particular A-scan was used as a reference signal while determining the size of interface delamination. Then the transmitterreceiver unit was moved to the $50 \mathrm{~mm}$ interface delamination region so that interface delamination was located between the transmitter and the receiver and another A-scan shown in Figure 6(b) was captured. It was observed in the experiments that the $\mathrm{S}_{0} \mathrm{~S}_{0}$ wave packet generated due to mode conversion has very low amplitude, compared to $\mathrm{S}_{0} \mathrm{~A}_{0}$ wave packet. Hence, the angle of the air-coupled receiver was adjusted in such a manner that it captures $\mathrm{S}_{0} \mathrm{~A}_{0}$ wave packet. This is the reason that we do not see $S_{0} S_{0}$ wave packet in Figure 6(b). The amplitude of $\mathrm{S}_{0} \mathrm{~A}_{0}$ wave packet generated due to delamination is $0.5 \mathrm{~V}_{\mathrm{pp}}$ as shown in Figure 6(b), which is higher when compared to $0.1 \mathrm{~V}_{\mathrm{pp}}$ when there is no delamination as shown in Figure 6(a). In the preliminary experiments, it was found that the attenuation of $S_{0}$ Lamb wave is higher when it travels from thin to thick region when compared thick to thin region. From the A-scans in Figure 6(a) and (b), the difference in arrival times of $\mathrm{S}_{0} \mathrm{~A}_{0}$ wave packet between a $50 \mathrm{~mm}$ delamination region and healthy region was found to be $30.4 \mu \mathrm{s}$. Using equation (3) delamination size from experiments is estimated to be $44.2 \mathrm{~mm}$, whereas the actual size of the interface delamination was $50 \mathrm{~mm}$. This shows that difference between predicted and measured value is less than $10 \%$. Small errors in time measurements are expected due to mechanical vibrations of $x-y$ scanner, which gives rise to positioning errors.

\section{Lamb wave-based B-scan imaging of interface delamination}

In the previous section, it was seen that when $\mathrm{S}_{0}$ mode interacts with a $\mathrm{T}$-joint with interface delamination it leads to generation of $\mathrm{S}_{0} \mathrm{~S}_{0}$ and $\mathrm{S}_{0} \mathrm{~A}_{0}$ wave packets. It is proposed here to estimate the size of delamination in the T-joint from the B-scan-based scanning technique. B-scan imaging consisted of capturing successive A-scan of mode-converted wave packets along a line by employing the air coupled as a receiver and exciting the T-joint structure with $\mathrm{S}_{0}$ mode. By collocating all the A-scans, a B-scan image can be obtained. Lamb wave-based B-scans were initially generated through numerical simulations in order to understand the propagating modes across the delamination using the same FE model discussed earlier and then compared with experimental B-scans.

\section{Receiver traversed from the thin to the thick region}

The initial and final positions of transmitter and receiver in numerical model and experiments are shown in Figure 7(a) and (b). The transmitter was excited at the edge of the T-joint on the thick region. The A-scans 


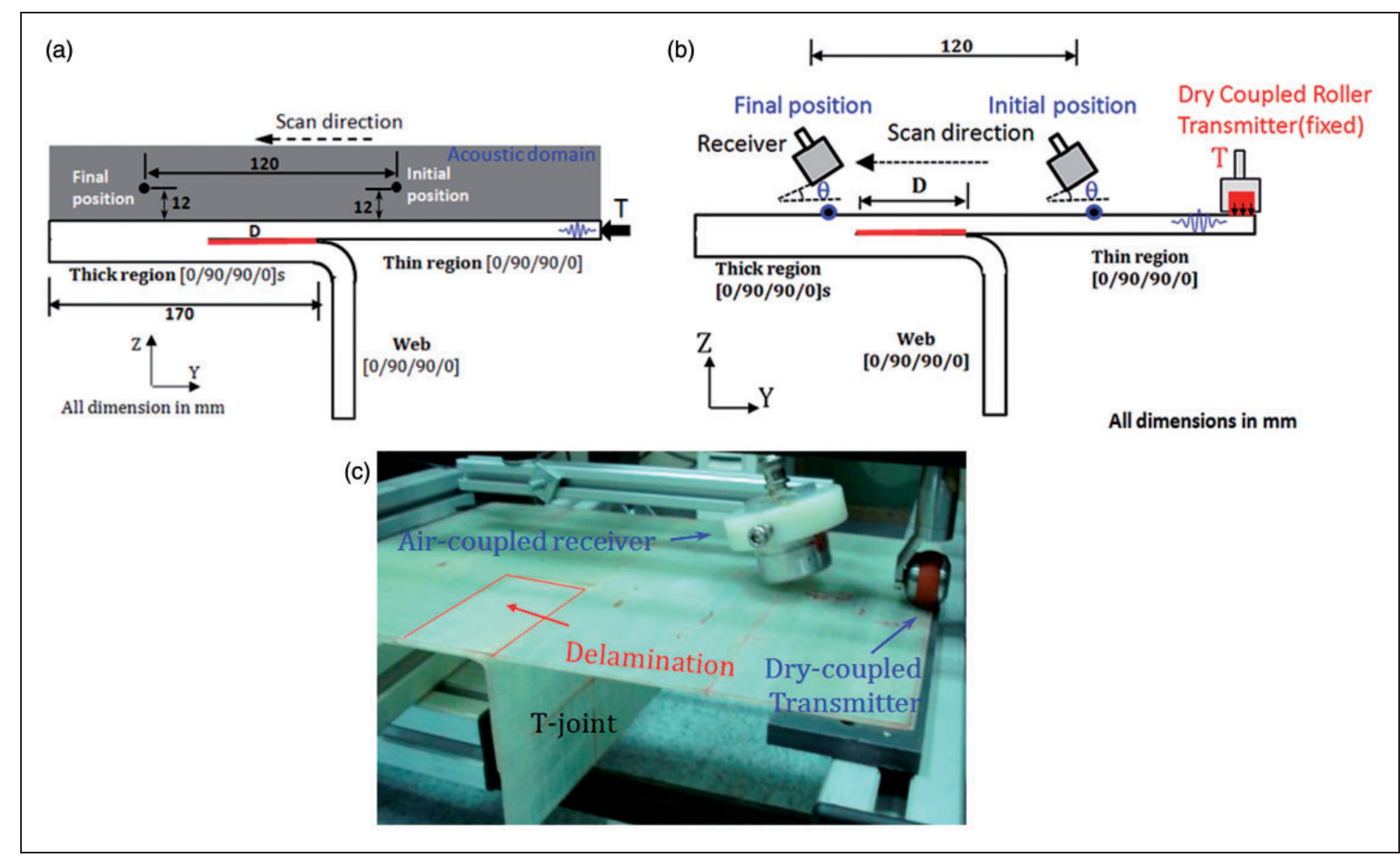

Figure 7. Schematic of the initial and final positions of the transmitter and receiver for generation of B-scans through (a) numerical model and (b) in the experiments, (c) experimental setup.

were captured in the acoustic domain traversing from the thin to the thick regions with a spatial resolution of $1 \mathrm{~mm}$. The initial position of the receiver was fixed on the thin region at a distance of $20 \mathrm{~mm}$ from the web. Then $100 \mathrm{~A}$-scans were captured in the thick region and 20 A-scans were captured in the thin region. A-scan data are normalized and stacked side by side to produce a B-scan using MATLAB program. The numerical B-scans were simulated with four different cases: (i) when there was no interface delamination, i.e. $\mathrm{D}=0$; (ii) $\mathrm{D}=40 \mathrm{~mm}$; (iii) $\mathrm{D}=50 \mathrm{~mm}$; and (iv) $\mathrm{D}=60 \mathrm{~mm}$ as shown in Figure 8(a) to (d), respectively. All B-scans are plotted in a colour scale where the low to high values are mapped from white to red with green indicating middle range values. In the B-scan, it can be observed that the incident $\mathrm{S}_{0}$ mode propagating towards the web location undergoes reflection and mode conversion due to geometrical discontinuity. The reflected waves travel in opposite direction. Comparison of the slopes of wave front after the web indicates generation of different modes. From all the numerical B-scans (Figure 8), it can be observed that $\mathrm{S}_{0} \mathrm{~A}_{0}$ wave packet has higher amplitude compared to $\mathrm{S}_{0} \mathrm{~S}_{0}$ wave packet. Additionally, depending on the width of delamination, the generation of $\mathrm{S}_{0} \mathrm{~A}_{0}$ wave packet is shifted further away from the web location compared to healthy region. In the B-scan of $50 \mathrm{~mm}$ delamination (Figure 8(c)), the $\mathrm{S}_{0} \mathrm{~A}_{0}$ generation point has shifted by $50 \mathrm{~mm}$ from the web location, compared to the B-scan at healthy region in Figure 8(a). This shift exactly corresponds to the width of delamination ' $D$ '. Similar evaluations were carried out for finding the widths of delaminations in B-scans shown in Figure $8(\mathrm{~b})$ to (d), and their predicted widths of delaminations match exactly with the actual values of 50 and $60 \mathrm{~mm}$, respectively.

\section{Receiver traversed from the thick to the thin region}

In this configuration one more set of numerical B-scans were generated by placing the transmitter and receiver at the thick and the thin regions, respectively. The A-scans were captured in the acoustic domain starting from the thick to the thin regions with a spatial resolution of $1 \mathrm{~mm}$. The maximum distance of separation at the end of the scanning between the transmitter and receiver was $200 \mathrm{~mm}$. B-scans were simulated at the healthy region and in the other three cases having 40 , 50 , and $60 \mathrm{~mm}$ width of interface delaminations, as shown in Figure 9(a) to (d), respectively. In all the four numerical B-scans, the initial position of the receiver was fixed at $100 \mathrm{~mm}$ from the web location on the thick region. In all the B-scans it was observed that due to mode conversion there is sudden drop in amplitude 


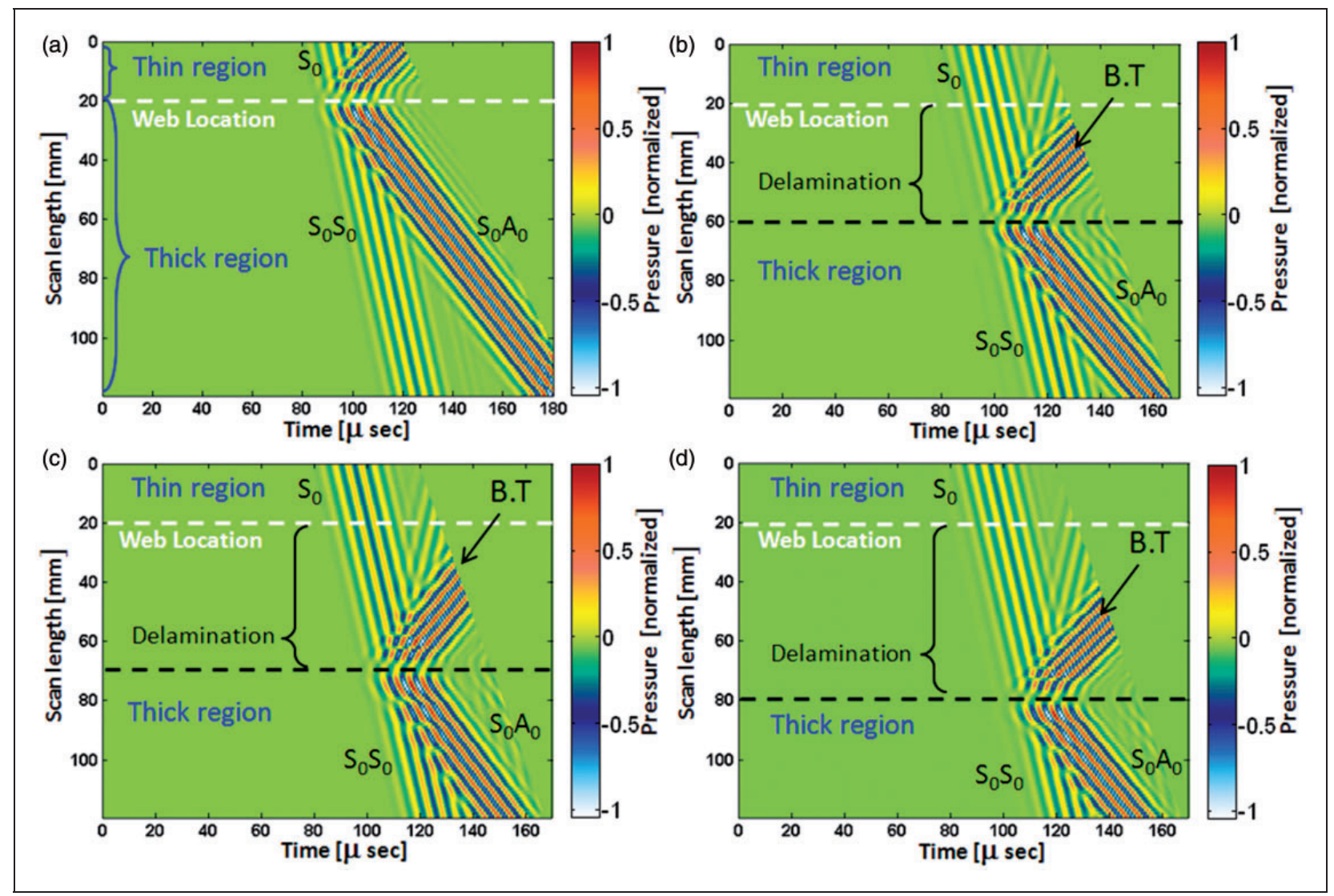

Figure 8. B-scans from numerical simulations showing the pressure distribution on top surface of the T-joint at (a) healthy region $(D=0)$, (b) $D=40 \mathrm{~mm}$, (c) $D=50 \mathrm{~mm}$, and (d) $D=60 \mathrm{~mm}$. The receiver was traversed from thin to thick region and the transmitter is excited in thin region. (Web location is shown by dotted white line, B.T (backward travelling wave)).

of about $6 \mathrm{~dB}$ of the $\mathrm{S}_{0} \mathrm{~S}_{0}$ wave packet, which exactly corresponds to the web location. Therefore, this feature can be used for prediction of the width of delamination. Depending on the starting point of delamination in the thick region, the $S_{0} S_{0}$ wave packet was generated further away from the web region. When the B-scan of $50 \mathrm{~mm}$ delamination, shown in Figure 9(c) is compared to the B-scan at healthy region shown in Figure 9(a), $\mathrm{S}_{0} \mathrm{~S}_{0}$ generation point was shifted by $50 \mathrm{~mm}$ from the web location, which is equal to the width of the delamination $(\mathrm{D}=50 \mathrm{~mm})$. Similarly, the widths of other delaminations in numerical B-scans shown in Figure 9(b) and (d) were found to be exactly matching with the actual value of 50 and $60 \mathrm{~mm}$ delamination, respectively.

\section{B-scans from experiments}

Experimental B-scans were generated by moving the air-coupled receiver over the T-joint and capturing successive A-scan while the dry-coupled transducer was fixed at the edge of the T-joint. The same experimental setup discussed earlier is used. The initial and final positions of the transmitter and receiver in experiments are shown in Figure 7(b). The angle of transducer was adjusted to either $4.5^{\circ}$ or $18^{\circ}$ (facing forward) to receive $\mathrm{S}_{0} \mathrm{~S}_{0}$ or $\mathrm{S}_{0} \mathrm{~A}_{0}$ wave packet, respectively. Experimental $\mathrm{B}$-scans were generated with similar transducer configuration discussed in the numerical simulation. In the B-scan shown in Figure 10(a) the transmitter was on the thin region and receiver was translated from thin to thick region. From the arrival times the $\mathrm{S}_{0} \mathrm{~A}_{0}$ wave packet was identified. To estimate the width of delamination, the differences in the point of generation of $\mathrm{S}_{0} \mathrm{~A}_{0}$ wave packet at healthy and defective region at the web were computed. However, compared to numerical B-scans, the differences in sizes of the regions in experimental B-scans are not equal to the actual width of delamination. This error essentially is due to finite size of the air-coupled receiver and small positioning errors due to mechanical vibrations of receiver during scanning. In the experimental B-scans shown in Figure 10(b), the size of the delamination region observed was $55 \mathrm{~mm}$ whereas the actual widths of delaminations were $50 \mathrm{~mm}$. It was found that oversizing could be discounted using half the receiver diameter 


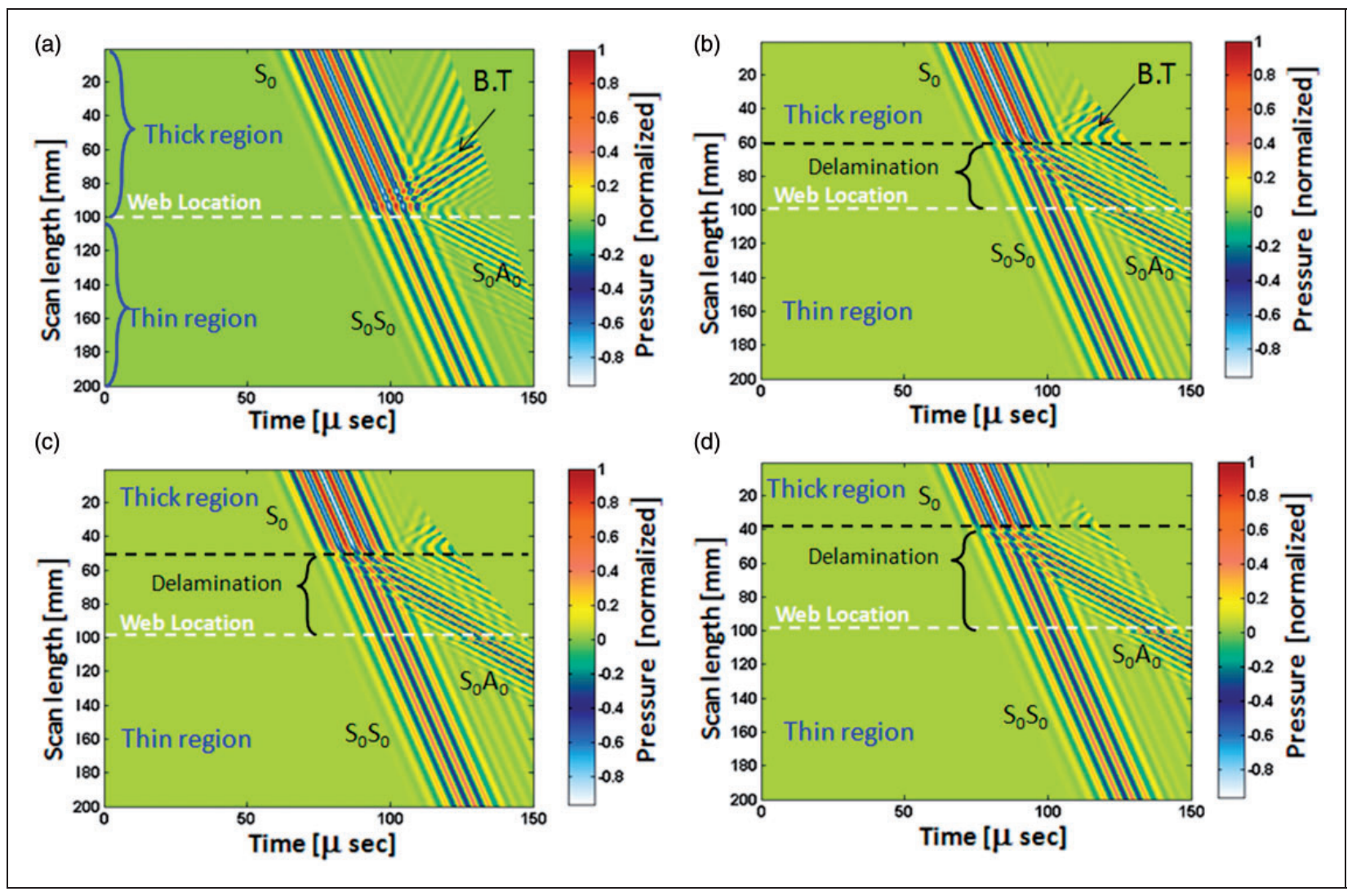

Figure 9. B-scan images from numerical simulations showing the pressure distribution on top surface of the T-joint at (a) healthy region ( $D=0$ ), (b) $D=40 \mathrm{~mm}$, (c) $D=50 \mathrm{~mm}$, and (d) $D=60 \mathrm{~mm}$. The receiver was traversed from thick to thin region and the transmitter is excited in thick region. (Web location is shown by dotted white line and B.T (backward travelling wave)).

(d/2), i.e. $(55-12.5=42.5 \mathrm{~mm})$, where ' $d$ ' is the diameter of the receiver. ${ }^{5}$

One more set of experimental B-scans were generated by placing the transmitter in the thick region. The receiver was translated from the thick to the thin regions with a spatial resolution of $1 \mathrm{~mm}$. The distance traversed by the receiver was $200 \mathrm{~mm}$. In this configuration, two experimental B-scans were generated: one at the healthy region and the other at $50 \mathrm{~mm}$ width of interface delaminations, as shown in Figure 11(a) and (b). The $\mathrm{S}_{0}$ mode travels from the thick region towards the thin region, where it is reflected, transmitted, and mode converted at the web region in case of a healthy $\mathrm{T}$-joint; whereas in case of T-joint with $50 \mathrm{~mm}$ interface delamination, mode conversion takes places at a much earlier location. During preliminary experiments, for this particular case it was found that when $\mathrm{S}_{0} \mathrm{~A}_{0}$ wave packet propagates through a distance of few centimetres, its amplitude reduces below the signal noise level and was not captured by air-coupled receiver. To overcome this problem, the air-coupled receiver was oriented at the $S_{0}$ mode angle of $4.5^{\circ}$ to receive $\mathrm{S}_{0} \mathrm{~S}_{0}$ wave packet. Furthermore, the B-scan shows that there is a rapid drop in amplitude of $S_{0}$ mode of about $6 \mathrm{~dB}$ due to mode conversion at the web location. Accordingly, based on the mode conversion and the amplitude loss, distinct regions can be differentiated from the experimental B-scan, which can be used as a feature for delamination identification. Consequently, the location of mode conversion should match with the start of delamination, which also coincides with the location of the generation of $\mathrm{S}_{0} \mathrm{~S}_{0}$ wave packet. Similar to the previous case, the sizes of the regions in experimental B-scans shown in Figure 11(a) and (b) do not match with the actual delamination size. This undersizing of the regions in the B-scans can be discounted with the half the size of the receiver $d / 2$ $(12.5 \mathrm{~mm})$. As a result, the estimated delamination width is $42+12.5=54.5 \mathrm{~mm}$. Thus, the predicted widths of delaminations from experiments were marginally higher compared to the actual size of $50 \mathrm{~mm}$. One of the main differences in the numerical B-scan and experimental B-scan images is due to material damping not accounted in simulation. Furthermore, in the experiments the air-coupled receiver is oriented to receive a specific mode and hence does not receive wave propagating in opposite direction such as the reflected wave at the delamination edge. 


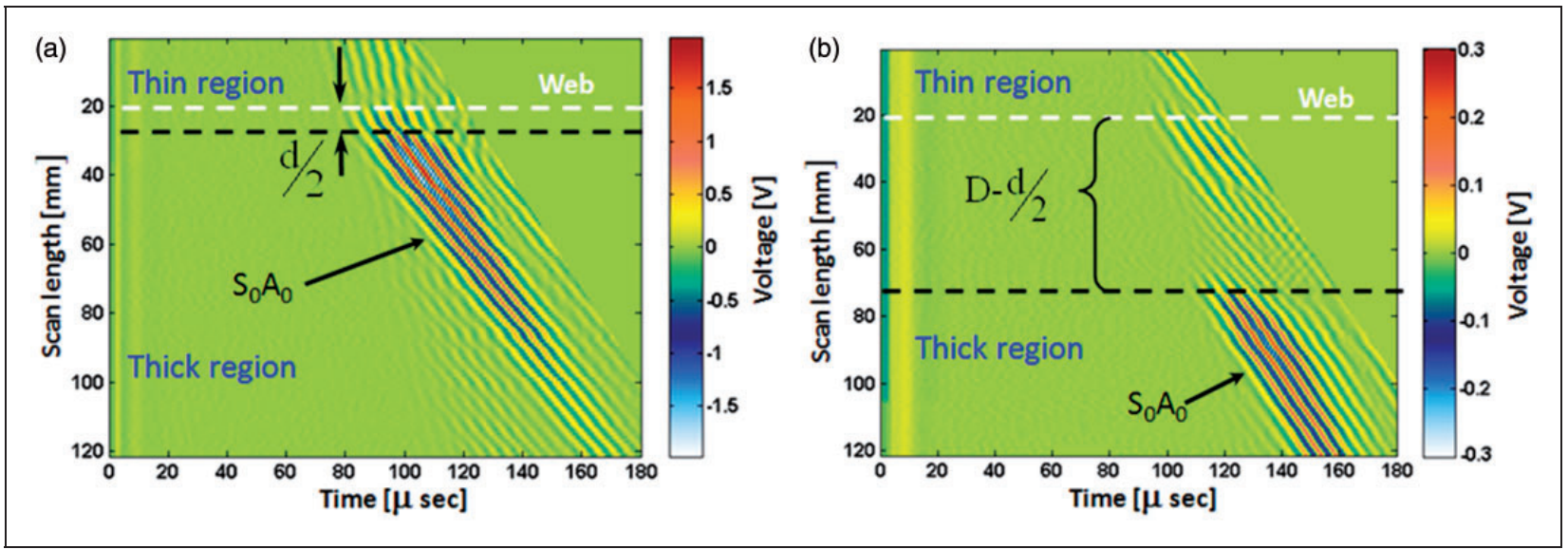

Figure 10. B-scans from experiments $(a)$ at healthy region $(D=0)$, and $(b)$ at $D=50 \mathrm{~mm}$. In this case, the air-coupled receiver is traversed from thin to thick region with the dry-coupled roller transmitter temporarily fixed in thin region.

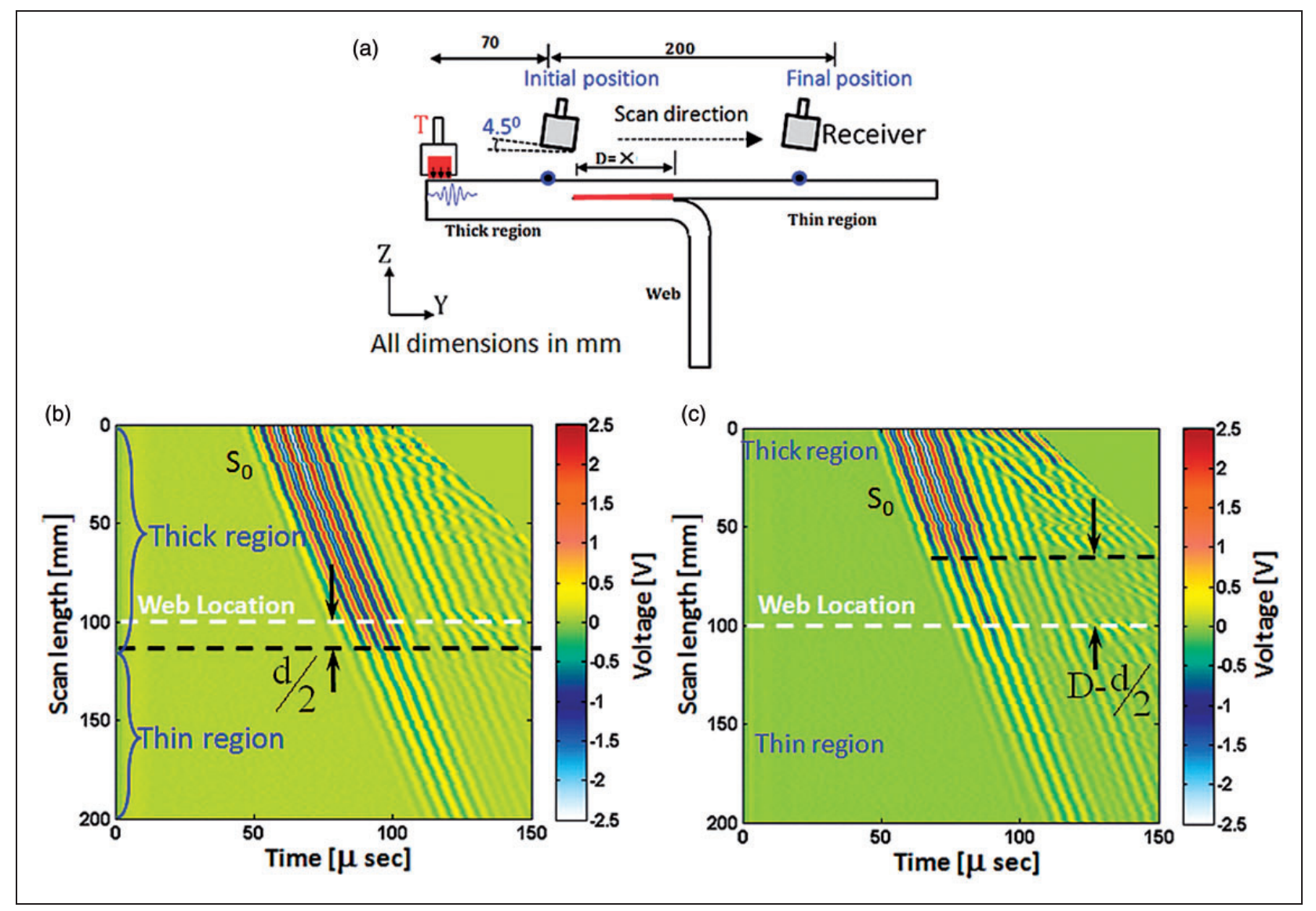

Figure II. (a) Schematic of the initial and final positions of transmitter and receiver. B-scans from experiments in the (b) healthy region $(D=0)$, (c) $D=50 \mathrm{~mm}$. In this case air-coupled receiver is traversed from thick to thin region and the dry-coupled roller transmitter is temporarily fixed in thick region. 


\section{Discussion and conclusion}

The interaction of fundamental symmetric $\mathrm{S}_{0}$ mode with a composite $\mathrm{T}$-joint was investigated using $\mathrm{FE}$ simulations followed by an experimental validation. From the FE simulations, it was observed that, when $\mathrm{S}_{0}$ mode interacts with the geometrical discontinuity, due to mode conversion $\mathrm{S}_{0} \mathrm{~S}_{0}$ and $\mathrm{S}_{0} \mathrm{~A}_{0}$ wave packet was generated in spite of any delamination. These wave packets propagate and transmit into the thin and the thick region. A novel technique was proposed to estimate the width of interface delamination in a $\mathrm{T}$-joint using arrival time of $\mathrm{S}_{0} \mathrm{~A}_{0}$ wave packet. In the experimental results, it was observed that the generated $\mathrm{S}_{0} \mathrm{~A}_{0}$ wave packet can be selectively captured by orienting the air-coupled transducer to $\mathrm{A}_{0}$ mode leakage angle either on top of thick or thin region. The numerical prediction of time of arrival of $\mathrm{S}_{0} \mathrm{~A}_{0}$ wave packet was found to be consistent with experimental results. One more method using Lamb wave-based B-scan imaging was proposed for predicting width of the interface delamination in the T-joint. Numerical B-scans revealed the fact that the sizes of the distinct regions in $\mathrm{B}$-scans depend on the $\mathrm{S}_{0}$ mode conversion to $\mathrm{S}_{0} \mathrm{~S}_{0}$ or $\mathrm{S}_{0} \mathrm{~A}_{0}$ wave packet. Two distinct regions were identified in each of the numerical B-scan and the sizes of the regions were found to be related to the width of delamination. Experimental B-scans also revealed the fact that the sizes of regions in B-scans also depend on diameter of the air-coupled receiver. B-scan regions were interpreted in terms of the width of the delamination and the diameter of the transmitter. Predicted values of the width of the delaminations were in agreement with the actual values after compensating with the diameter of the air-coupled receiver. Thus, both methods were found effective for quantitative detection and sizing of the delamination. The experimental results successfully demonstrate usefulness of the liquid couplant-free configuration to excite and receive Lamb waves in the T-joint.

Even though the experiments performed in this study are on a simple $\mathrm{T}$-joint and fundamental in nature, these results presented can be generalized provided the mode-converted $\mathrm{S}_{0} \mathrm{~S}_{0}$ or $\mathrm{S}_{0} \mathrm{~A}_{0}$ wave packet is of sufficient measurable amplitude. The liftoff between an air-coupled receiver and the structure must be constant throughout the entire measurement process, so that accurate time of arrival can be estimated. Conversely, this may not be difficult to maintain in an automated scanner. When the Lamb wave propagates from thick to thin region and vice versa, it was observed that the mode conversion created by geometrical discontinuity is often lower in amplitude and waves transmitted from delamination rapidly die out. Hence, care should to be taken while selecting source-receiver distances during scanning operation. Nevertheless, this B-scan technique proposed has a potential to be used as quick inspection of stiffened-skin structures.

\section{Acknowledgements}

The authors thank Dr Ramadas Chennamsetti from R\&DE(E), DRDO and Dr Mahesh Bhardwaj from Ultran Group for the provision of GMP based air-coupled transducers. The authors acknowledge R\&DE(E), DRDO, Pune for providing the $\mathrm{T}$-joint sample.

\section{Conflict of interest}

The author(s) declared no potential conflicts of interest with respect to the research, authorship, and/or publication of this article.

\section{Funding}

The author(s) disclosed receipt of the following financial support for the research, authorship, and/or publication of this article:

This research received funding from the NPMASS program managed by ADA, Bangalore.

\section{References}

1. Su Z, Ye L and Lu Y. Guided Lamb waves for identification of damage in composite structures: a review. J Sound Vib 2006; 295: 753-780.

2. Greve DW, Tyson $\mathbf{N}$ and Oppenheim IJ. Interaction of defects with Lamb waves in complex geometries. IEEE Ultrasonics Conf Proc 2005; 297-300.

3. Reusser RS, Chimenti DE, Roberts RA, et al. Guided plate wave scattering at vertical stiffeners and its effect on source location. Ultrasonics 2012; 52: 687-693.

4. Yeum CM, Sohn H, Ihn JB, et al. Instantaneous delamination detection in a composite plate using a dual piezoelectric transducer network. Compos Struct 2012; 94: 3490-3499.

5. Ramadas C, Janardhan P, Balasubramaniam K, et al. Lamb wave based ultrasonic imaging of interface delamination in a composite T-joint. NDT\&E Int 2011; 44: 523-530.

6. Castaings $\mathrm{M}$ and Cawley $\mathrm{P}$. The generation, propagation, and detection of Lamb waves in plates using air-coupled ultrasonic transducers. J Acoust Soc Am 1996; 100: 3070-3077.

7. Ramadas C, Balasubramaniam K, Joshi $M$, et al. Numerical and experimental studies on propagation of $\mathrm{A}_{0}$ mode in a composite plate containing semi-infinite delamination: observation of turning modes. Compos Struct 2011; 93: 1929-1938.

8. Demcenko A, Zukauskas E, Kazys R, et al. Interaction of the $\mathrm{A}_{0}$ Lamb wave mode with a de-lamination type defect in GLARE 3-3/2 composite material. Acustica-Acta Acustica 2006; 92: 540-549.

9. Ramadas C, Balasubramaniam K, Joshi M, et al. Interaction of the primary anti-symmetric Lamb mode $\left(\mathrm{A}_{0}\right)$ with symmetric delaminations: numerical and experimental studies. Smart Mater Struct 2009; 18: 085011s. 
10. Kazys R, Demcenko A, Zukauskas E, et al. Air-coupled ultrasonic investigation of multi-layered composite materials. Ultrasonics 2006; 44: 819-822.

11. Raisutis R, Kazys R, Zukauskas E, et al. Ultrasonic aircoupled testing of square-shape CFRP composite rods by means of guided waves. NDT\&E Int 2011; 44: 645-654.

12. Mustapha S, Lin Y, Dong W, et al. Assessment of debonding in sandwich $\mathrm{CF} / \mathrm{EP}$ composite beams using $\mathrm{A}_{0}$ Lamb wave at low frequency. Compos Struct 2011; 93: 483-491.

13. Guo $\mathrm{N}$ and Cawley P. The interaction of Lamb waves with delaminations in composite laminates. $J$ Acoust Soc Am 1993; 94: 2240-2246.

14. Hayashi $\mathrm{T}$ and Kawashima K. Multiple reflections of Lamb waves at a delamination. Ultrasonics 2002; 40: 193-197.

15. $\mathrm{Hu} \mathrm{N}$, Shimomukai T, Yan C, et al. Identification of delamination position in cross-ply laminated composite beams using $\mathrm{S}_{0}$ Lamb mode. Compos Sci Technol 2008; 68: 1548-1554.

16. Tan KS, Guo N, Wong BS, et al. Comparison of Lamb waves in pulse echo detection of near-surface defects in laminate plates. NDT\&E Int 1995; 28: 215-223.

17. Toyama N, Noda J and Okabe T. Quantitative damage detection in cross-ply laminates using Lamb wave method. Comp Sci Technol 2003; 63: 1473-1479.

18. Koh YL, Chiu WK and Rajic N. Integrity assessment of composite repair patch using propagating Lamb waves. Compos Struct 2002; 58: 363-371.

19. Pan N, Su Z, Ye L, et al. A quantitative identification approach for delamination in laminated composite beams using digital damage fingerprints (DDFs). Compos Struct 2006; 75: 559-570.

20. Ip KH and Mai YW. Delamination detection in smart composite beams using Lamb waves. Smart Mater Struct 2004; 13: 544-551.

21. Rosalie SC, Vaughan M, Bremner A, et al. Variation in the group velocity of Lamb waves as a tool for the detection of delamination in GLARE aluminium plate-like structures. Compos Struct 2004; 66: 77-86.

22. Petculescu G, Krishnaswamy S and Achenbach JD. Group delay measurements using modally selective Lamb wave transducers for detection and sizing of delaminations in composites. Smart Mater Struct 2007; 17: 015007.
23. Wang D, Ye L, Lu Y, et al. Probability of the presence of damage estimated from an active sensor network in a composite panel of multiple stiffeners. Compos Sci Technol 2009; 69: 2054-2063.

24. Yang $M$ and Qiao P. Modeling and experimental detection of damage in various materials using the pulse-echo method and piezoelectric sensors/actuators. Smart Mater Struct 2005; 14: 1083-1100.

25. Takeda S, Okabe Y and Takeda N. Delamination detection in CFRP laminates with embedded small-diameter fiber Bragg grating sensors. Compos Part A 2000; 33: 971-980.

26. Sohn H, Dutta D, Yang JY, et al. Delamination detection in composites through guided wave field image processing. Compos Sci Technol 2011; 71: 1250-1256.

27. Pierce SG, Culshaw B, Manson G, et al. Application of ultrasonic Lamb wave techniques to the evaluation of advanced composite structures. In: SPIE 7th Annual Int Symp on Smart Struct Mater 2000, pp.93-103.

28. Yashiro S, Takatsubo J and Toyama N. An NDT technique for composite structures using visualized Lamb wave propagation. Compos Sci Technol 2007; 67: 3202-3208.

29. Michaels TE, Michaels JE, Mi B, et al. Damage detection in plate structures using sparse ultrasonic transducer arrays and acoustic wave field imaging. AIP Rev Prog Quant Nondestr Eval 2005; 24A: 938-945.

30. Hillger $W$ and Szewieczek A. Visualization of guided wave propagation by ultrasonic imaging methods. Int $J$ Mater Prod Technol 2011; 41: 1-4.

31. Hillger W, Szewieczek A and Schmidt D. Damage detection in a helicopter composite tailboom by mode conversion of Lamb waves. In: Sixth European Workshop on Structural Health Monitoring, Dresden, Germany, 2012.

32. Szewieczek A, Heinze C, Hillger W, et al. Analysis methods of lamb wave propagation in complex composites. In: Sixth European Workshop on Structural Health Monitoring, Dresden, Germany, 2012.

33. DISPERSE Software version 2.0.16b. Imperial College, London, UK, 2003.

34. Yang C, Lin Ye, Su Z, et al. Some aspects of numerical simulation for Lamb wave propagation in composite laminates. J Compos Struct 2006; 75: 267-275. 


\title{
Quantitative characterization of interface delamination in composite T-joint using couplant-free Lamb wave methods
}

\author{
Padiyar M, Janardhan
}

Sage

Padiyar MJ, Balasubramaniam K. (2016) Quantitative characterization of interface delamination in composite T-joint using couplant-free Lamb wave methods. Journal of Reinforced Plastics and Composites, Volume 35, Issue 4, February 2016, pp.345-361

https://doi.org/10.1177/0731684415617247

Downloaded from Cranfield Library Services E-Repository 